\title{
Characterization of Qubit Dephasing by Landau-Zener-Stückelberg-Majorana Interferometry
}

\author{
F. Forster, ${ }^{1}$ G. Petersen, ${ }^{1}$ S. Manus, ${ }^{1}$ P. Hänggi, ${ }^{2}$ D. Schuh,${ }^{3}$ W. Wegscheider,${ }^{3,4}$ S. Kohler, ${ }^{5}$ and S. Ludwig ${ }^{1}$ \\ ${ }^{1}$ Center for NanoScience \& Fakultät für Physik, LMU-Munich, 80539 München, Germany \\ ${ }^{2}$ Institut für Physik, Universität Augsburg, 86135 Augsburg, Germany \\ ${ }^{3}$ Fakultät für Physik, Universität Regensburg, 93040 Regensburg, Germany \\ ${ }^{4}$ Solid State Physics Laboratory, ETH Zurich, 8093 Zurich, Switzerland \\ ${ }^{5}$ Instituto de Ciencia de Materiales de Madrid, CSIC, 28049 Madrid, Spain
}

(Received 5 December 2013; published 19 March 2014)

\begin{abstract}
Controlling coherent interaction at avoided crossings and the dynamics there is at the heart of quantum information processing. A particularly intriguing dynamics is observed in the Landau-Zener regime, where periodic passages through the avoided crossing result in an interference pattern carrying information about qubit properties. In this Letter, we demonstrate a straightforward method, based on steady-state experiments, to obtain all relevant information about a qubit, including complex environmental influences. We use a two-electron charge qubit defined in a lateral double quantum dot as test system and demonstrate a long coherence time of $T_{2} \simeq 200 \mathrm{~ns}$, which is limited by electron-phonon interaction.
\end{abstract}

DOI: 10.1103/PhysRevLett.112.116803

PACS numbers: 73.63.Kv, 03.65.Yz, 03.67.-a

A qubit is a quantum mechanical two-level system characterized by its tunnel coupling and the detuning from its symmetry point at which the qubit levels form an avoided crossing. Dynamic control can be achieved by switching the detuning between finite values and zero at velocities ranging from almost sudden to adiabatically slow changes. A particularly intriguing dynamics was predicted by Landau, Zener, Stückelberg, and Majorana (LZSM) [1-4] for the intermediate (Landau-Zener) regime that is hallmarked by quantum superpositions. These give rise to interference for the case of periodic passages [5-11]. A particular application is LZSM interferometry, a double-slit kind of experiment that, in principle, can be realized with any qubit, while the specific measurement protocol might vary. Ours is based on two-electron states in a lateral double quantum dot (DQD) embedded in a two-dimensional electron system (2DES) (Fig. 1). Source and drain leads at chemical potentials $\mu_{S, D}$, each tunnel coupled to one dot, allow current flow by single-electron tunneling. Applying the voltage $V=\left(\mu_{S}-\mu_{D}\right) / e=1 \mathrm{mV}$ across the DQD [Fig. 1(b)], we use this current to detect the steady-state properties of the driven system. We take the singlet $S_{11}$ (one electron in each dot) and the singlet $S_{20}$ (two electrons in the left dot) as charge qubit states. They form an avoided crossing [Fig. 1(c)], described by the Hamiltonian

$$
H_{\text {qubit }}=\left(\begin{array}{cc}
0 & \Delta / 2 \\
\Delta / 2 & -\varepsilon(t)
\end{array}\right)
$$

where we consider a variable energy detuning $\varepsilon(t)$ and a constant interdot tunnel coupling tuned to $\Delta \simeq 13 \mu \mathrm{eV}$, corresponding to a clock speed of $\Delta / h \simeq 3.1 \mathrm{GHz}$, where $h$ is the Planck constant.
Let us first discuss a single sweep through the avoided crossing at $\varepsilon=0$ : as shown back in 1932 independently by Landau, Zener, Stuickelberg, and Majorana, it brings the qubit into a superposition state [1-4], the electronic analog to the optical beam splitter [13-17]. The probability to remain in the initial qubit state $P_{\mathrm{LZ}}=\exp \left(-\pi \Delta^{2} / 2 \hbar v\right)$ thereby grows with the velocity $v=d \varepsilon / d t$, here assumed to be constant [1-4]. Because the relative phase between the split wave packets depends on their energies, repeated passages by a periodic modulation $\varepsilon(t)=\bar{\varepsilon}+A \cos (\Omega t)$ give rise to so-called LZSM quantum interference [5-11]. We present a breakthrough that makes LZSM interferometry a powerful tool: it is based on systematic measurements together with a realistic model, which explicitly includes the noisy environment. We demonstrate how to decipher the detailed qubit dynamics and directly determine its decoherence time $T_{2}$ based on straightforward steadystate measurements.

Keeping the experiment simple, we detect the dc-current $I$ through the DQD. It involves electron tunneling giving rise to the configuration cycle $(1,0) \rightarrow(1,1) \leftrightarrow(2,0) \rightarrow(1,0)$, where pairs of digits refer to the number of electrons charging the (left, right) dot [Fig. 1(b)]. The energetically accessible two-electron states include the singlets $S_{11}$ and $S_{20}$ but also three triplets $T_{11}$ [Figs. 1(b), 1(c)]. These triplets are likely occupied during $(1,0) \rightarrow(1,1)$, and their decay via a spin flip $T_{11} \rightarrow S_{11}$ is hindered by a Pauli-spin blockade $[18,19]$. This slows down the transition $(1,1) \rightarrow$ $(2,0)$ and thereby limits the current. To nevertheless quickly initialize the qubit and generate a measurable current, we lift the blockade using an on-chip nanomagnet [Fig. 1(a)] [12]. $I$ is proportional to the occupation probability of $S_{20}$ and serves as destructive qubit detector. 


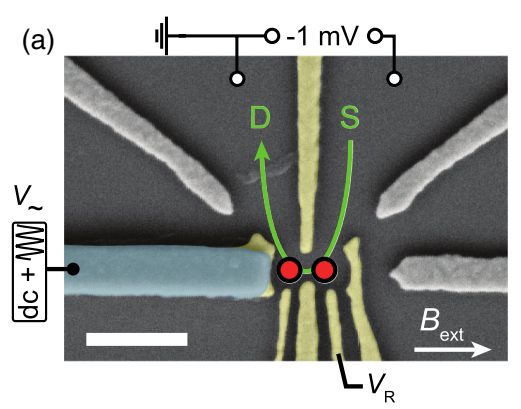

(b)

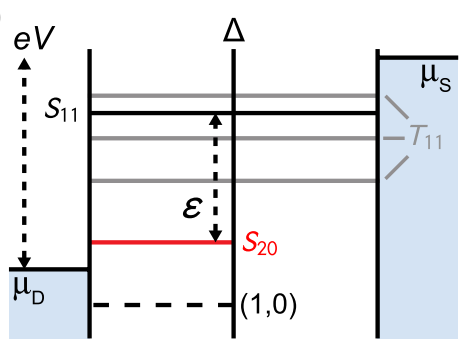

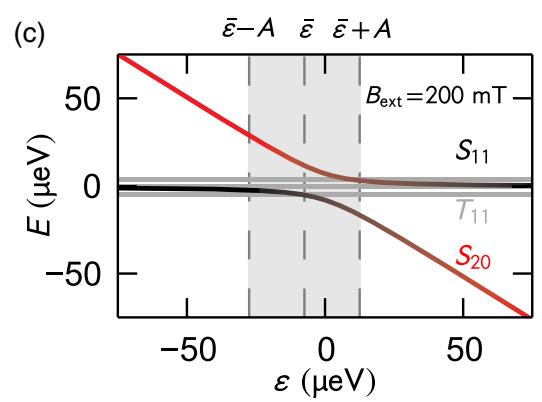

FIG. 1 (color online). Experimental setup. (a) Scanning electron micrograph showing Ti/Au gates, fabricated by electron-beam lithography, on the surface of a GaAs/AlGaAs heterostructure, grown by molecular beam epitaxy ( $500 \mathrm{~nm}$ scale bar). $85 \mathrm{~nm}$ beneath the surface it contains a 2DES with carrier density $n_{\mathrm{e}}=1.19 \times 10^{11} \mathrm{~cm}^{2}$ and mobility $\mu=0.36 \times 10^{6} \mathrm{~cm}^{2} \mathrm{~V}^{-1} \mathrm{~s}^{-1}$. Six of the Ti/Au gates (light yellow) are biased with negative voltages to electrostatically define a DQD in the 2DES, and the other gates are grounded. A cobalt single-domain nanomagnet (thick blue bar) produces an inhomogeneous magnetic field that slightly mixes singlet and triplet states of the DQD [12]. (b) Typical situation in our two-electron DQD: vertical lines indicate tunable tunnel barriers, horizontal lines mark the chemical potentials, blue areas are the degenerate 2DES leads. The voltage $V=\left(\mu_{S}-\mu_{D}\right) / e$ causes a single-electron tunneling current [green arrow in panel (a)]. (c) Energy diagram of the relevant two-electron DQD eigenstates. Singlets (the qubit states) are represented as black and red lines; triplets, which are Zeeman split, are represented as gray lines. Rf modulation of the gate voltage $V_{\sim}$ [panel (a)] results in a modulated detuning $\varepsilon(t)$, indicated by gray shading.

As it is possible to tune the relative couplings and the mean detuning $\bar{\varepsilon}$ of the singlet-singlet and singlet-triplet crossings by gate voltages and magnetic fields, our two-electron DQD opens two interesting perspectives: (i) LZSM interferometry involving multiple avoided crossings [8,20-23] and (ii) coherent Landau-Zener transitions between our charge qubit and the recently very successful spin-based qubits [24]; see the Supplemental Material [25], Sect. IIA for details.

Concentrating on the two-electron charge qubit, in Figs. 2(a) and 2(b) we display LZSM interference patterns measured at $T_{2 \mathrm{DES}} \simeq 20 \mathrm{mK}$ for two different modulation frequencies $\Omega / 2 \pi$. Within the triangle defined by $A \gtrsim|\bar{\varepsilon}|$, the qubit is periodically driven through the avoided crossing and the current oscillates between zero and distinct maxima indicating destructive and constructive interference $[9,26]$. An interpretation based on photon-assisted tunneling, which is for $\hbar \Omega \gtrsim \Delta$ fully equivalent to the LZSM picture discussed above, facilitates quantitative predictions: using Floquet scattering theory [27], we find for $\Delta \ll \hbar \Omega$ the current per spin projection

$$
I(\bar{\varepsilon}, A)=\frac{e}{\hbar} \frac{\Gamma_{\mathrm{in}} \Gamma_{\text {out }}}{4 \gamma} \sum_{n=-\infty}^{\infty} \frac{\Delta_{n}^{2}}{(\bar{\varepsilon}-n \hbar \Omega)^{2}+\Delta_{n}^{2}+\gamma^{2}},
$$

where $\Gamma_{\text {in }}$ is the qubit initialization rate $(1,0) \rightarrow S_{11}, \Gamma_{\text {out }}$ is the decay rate $(2,0) \rightarrow(1,0)$, and $\gamma=(1 / 2)\left(\Gamma_{\text {in }}+\Gamma_{\text {out }}\right)$.

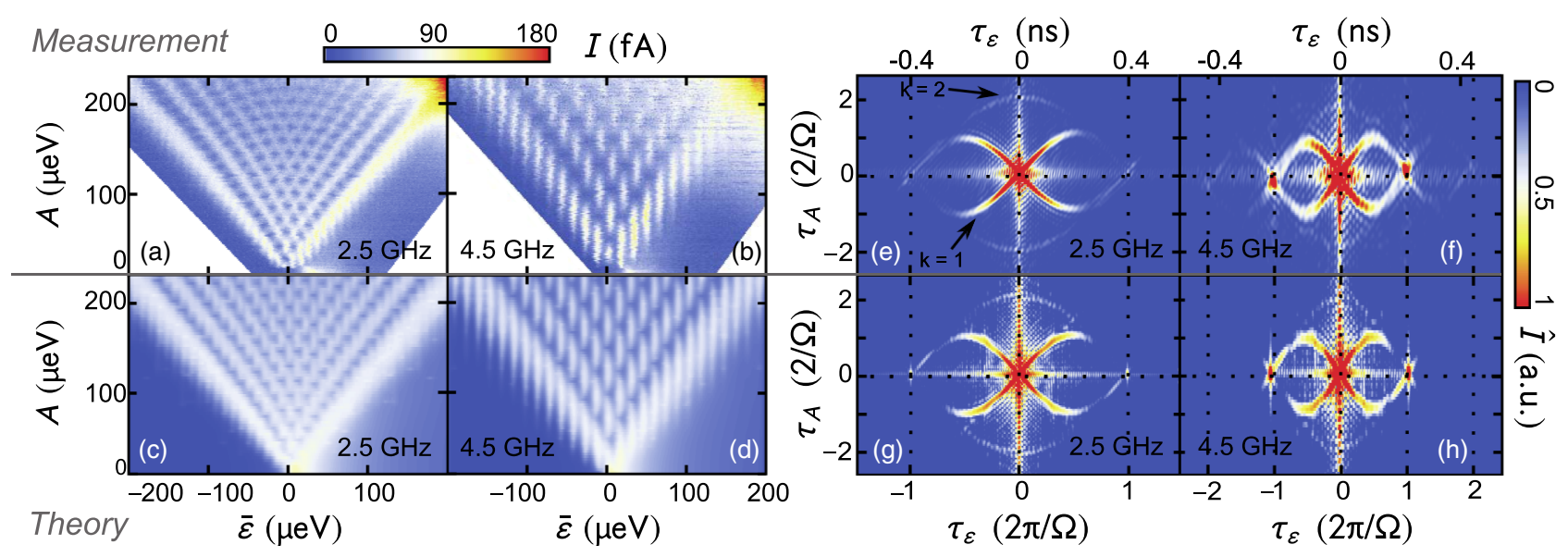

FIG. 2 (color online). LZSM interference. Measured current through the DQD as a function of mean detuning $\bar{\varepsilon}$ and modulation amplitude $A$ at $T \simeq 20 \mathrm{mK}$ for the modulation frequencies $2.5 \mathrm{GHz}$ (a) and $4.5 \mathrm{GHz}$ (b). [(c), (d)] Corresponding numerically calculated current for realistic conditions. [(e)—(h)] Two-dimensional numerical Fourier transformed $\left(A \rightarrow \tau_{A}, \bar{\varepsilon} \rightarrow \tau_{\varepsilon}, I \rightarrow \hat{I}\right)$ of measurements (upper panels) and theory (lower panels). The shape of the sinusoidal branches of enhanced $\hat{I}$ is determined by $\Omega$; see Eq. (3). Their decay with increasing $\tau_{\varepsilon}$ encodes dephasing and decoherence. The horizontal and vertical lines of enhanced amplitude at $\tau_{A}=0$ and $\tau_{\varepsilon}=0$ are artifacts caused by the finite region of data being transformed. 
The interdot tunnel coupling is renormalized with the $n$ thorder Bessel function $J_{n}$ of the first kind: $\Delta_{n}=J_{n}(A / \hbar \Omega) \Delta$. Equation (2) predicts Lorentz-shaped current maxima of width $\delta \bar{\varepsilon}=\sqrt{\Delta_{n}^{2}+\gamma^{2}}$ at $\bar{\varepsilon}=n \hbar \Omega$, which for $\Delta \ll|\bar{\varepsilon}|$ corresponds to the bare $n$-photon resonance $\sqrt{\bar{\varepsilon}^{2}+\Delta^{2}}=$ $n \hbar \Omega$. The peaks are modulated by $J_{n}^{2}(A / \hbar \Omega)$ as a function of $A$. This scattering approach provides an appealing physical picture and describes the main features of the measured LZSM patterns as can be easily seen for the high-frequency limit $\hbar \Omega \gg \delta \bar{\varepsilon}$ (see the Supplemental Material [25], Fig. 5). For lower $\Omega$, the distance between current peaks is smaller and, hence, the broadened resonances tend to merge [Fig. 2(a)].

The visibility of the LZSM pattern (i) depends on frequency and amplitude via the Landau-Zener probability $P_{\mathrm{LZ}}$ [captured in Eq. (2) by $\Delta_{n}$ ], (ii) is strongest for $\Gamma_{\text {in }} \simeq \Gamma_{\text {out }}$, and (iii) is diminished for $\Delta<\gamma$, where the qubit decay is faster than its clock speed. However, Eq. (2) fails to predict the qubit coherence time as it ignores environmental noise. The nevertheless qualitative consent indicates that environmental noise can be treated perturbatively. In this spirit, we developed a complete model that goes beyond Eq. (2) by explicitly including all energetically accessible states of our driven DQD and, importantly, decoherence within a system-bath approach.

An evident source of decoherence is the interaction of the qubit electrons with bulk phonons [28], which entails quantum fluctuations to the DQD level energies. It enters our theory as dissipation kernel with a dimensionless electron-phonon coupling strength $\alpha_{Z}$ (see the Supplemental Material [25], Sect. III) derived from a system-bath approach, becoming the spin-boson model in the qubit subspace [29]. We assume for the coupling an Ohmic spectral density, which is justified by geometry considerations (see the Supplemental Material [25], Sect. III C) and also a posteriori by a surprisingly good agreement with our experimental results.

The second environmental component of our model is charge noise, well known to cause low-frequency fluctuations of the local confinement potential in semiconductor heterostructures [30,31]. Being slow compared to all relevant time scales of our experiment, they can be treated as static disorder leading in the ensemble average to an inhomogeneous, Gaussian broadening of width $\lambda^{\star}$.

To determine the key parameters $\lambda^{\star}$ and $\alpha_{Z}$, we compare our measurements with theoretical results obtained within a Bloch-Redfield master equation which can be solved efficiently after a decomposition into the Floquet basis of the rf-driven DQD (see Supplemental Material [25], Sect. IV). The optimized result is displayed in Figs. 2(c) and 2(d) with $\lambda^{\star}=3.5 \mu \mathrm{eV}$ and $\alpha_{Z}=1.5 \times 10^{-4}$. Below, we illustrate the self-consistent fit procedure by first determining $\lambda^{\star}$ based on the final value of $\alpha_{Z}$ and then evaluating $\alpha_{Z}$ using the final value of $\lambda^{\star}$.

Figure 3(a) displays $I(\bar{\varepsilon})$ for $\Omega / 2 \pi=2.75 \mathrm{GHz}$ and constant amplitude $A$, corresponding to a horizontal slice in the presentations of Figs. 2(a)-2(d). The measured data (dots) in Fig. 3(a) feature a beating of broadened and overlapping current peaks. The gray line is calculated for $\alpha_{Z}=1.5 \times 10^{-4}$ and $\lambda^{\star}=0$. Compared to our measurement, it shows a weaker broadening and a higher visibility. Much better agreement is reached for $\lambda^{\star}=3.5 \mu \mathrm{eV}$ (blue line). This result is robust under moderate variations of $\alpha_{Z}$ and does not depend on frequency or temperature. Figure 3(b) underlines the good agreement between measured (dots) versus calculated (lines) data by presenting $I(A)$ at $\bar{\varepsilon}=n \hbar \Omega$ for various $n$ [vertical slices in Figs. 2(a)-2(d)]. Owing to the electron-phonon interaction, the visibility of the interference pattern drops with increasing temperature [Fig. 3(c)].

To quantify $\alpha_{Z}$ with high accuracy, we use this temperature dependence and thereby capture global information of the extended LZSM patterns [Figs. 2(a)-2(d)] by performing two-dimensional Fourier transformations $I(\bar{\varepsilon}, A) \rightarrow \hat{I}\left(\tau_{\varepsilon}, \tau_{A}\right)$. The results, featured in Figs. 2(e)-2(h), are simple, lemonshaped structures of local maxima $\left.\hat{I}\left(\tau_{\varepsilon}, \tau_{A}\right)\right|_{\text {lemon }}$. Transforming Eq. (2) yields an analytic formula describing these lemon arcs

$$
\tau_{A}= \pm \frac{2 k}{\Omega} \sin \left(\frac{\Omega \tau_{\varepsilon}+2 \pi k^{\prime}}{2 k}\right)
$$

with $k=1,2,3, \ldots, k^{\prime}=0,1,2, \ldots$, and $k^{\prime}<k$. Arcs for $k>1$ are a consequence of $\Delta \gtrsim \gamma$, a prerequisite for
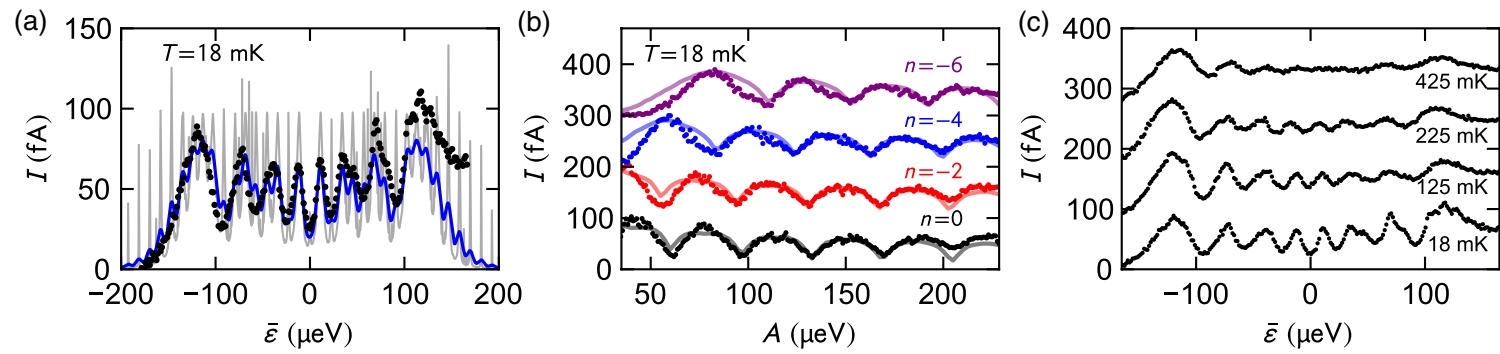

FIG. 3 (color online). Raw data analysis. Dots are measured at $\Omega / 2 \pi=2.75 \mathrm{GHz}$, lines numerical data for $\alpha_{Z}=1.5 \times 10^{-4}$ and $\lambda^{\star}=3.5 \mu \mathrm{eV}$, whereas the gray line in panel (a) is for $\lambda^{\star}=0$. (a) Horizontal slice through a LZSM pattern: $I(\bar{\varepsilon})$ for a constant $A=130 \mu \mathrm{eV}$. (b) Vertical slices through a LZSM pattern: $I(A)$ for $\bar{\varepsilon} / \hbar \Omega=0,-2,-4,-6$. (c) Measured data as in panel (a) for various temperatures. 

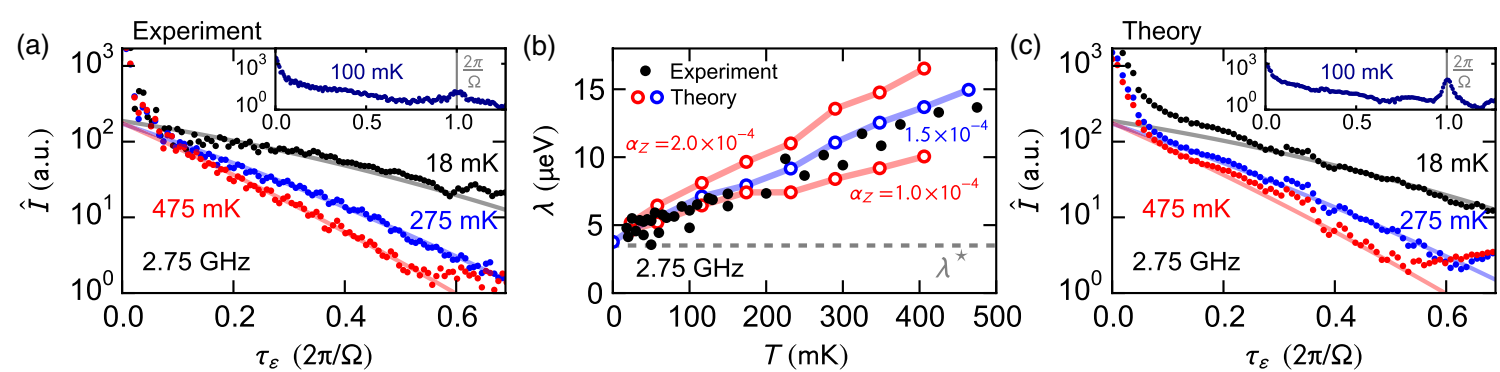

FIG. 4 (color online). Electron-phonon coupling. (a) Decaying region of the measured $\left.\hat{I}\left(\tau_{\varepsilon}, \tau_{A}\right)\right|_{\text {lemon }}$ for three temperatures (dots). Lines are generated using Eq. (4) for $\lambda^{\star}=3.5 \mu \mathrm{eV}$ and $\lambda$ as a fit parameter. The inset shows a broader region including maxima at $\tau_{\varepsilon}=0,2 \pi / \Omega$. (b) Measured decay rate $\lambda(T)$ (black dots) and corresponding numerical data (colored circles) based on $\lambda^{\star}=3.5 \mu \mathrm{eV}$ (indicated as horizontal line) and $\alpha_{Z}=1.0,1.5,2.0 \times 10^{-4}$. (c) Analog to panel (a) but based on numerical calculations. Solid lines are identical to those in (a). The numerical resolution is based on 100 data points sampling the Gaussian broadening in $\bar{\varepsilon}$ of width $\lambda^{\star}=3.5 \mu \mathrm{eV}$.

observing a pronounced interference pattern (see the Supplemental Material [25], Sect. II.E). [Arcs for $k>1$ are weakly seen in Figs. 2(e)-2(h). In superconducting qubits they have been also observed but-considering $\Delta \ll \gamma$ [32]-not explained.] Concentrating on the principal lemon arc for $k=1$, we find a nonmonotonic behavior of $\left.\hat{I}\left(\tau_{\epsilon}, \tau_{A}\right)\right|_{\text {lemon }}$ with maxima at the arc's intersections at $\tau_{A}=0$ and $\tau_{\varepsilon}$ a multiple of $2 \pi / \Omega$. Regions of decays in between have the form

$$
\left.\hat{I}\left(\tau_{\varepsilon}, \tau_{A}\right)\right|_{\text {lemon }} \propto e^{-\lambda\left|\tau_{\varepsilon}\right| / \hbar} e^{-\frac{1}{2}\left(\lambda^{*} \tau_{\varepsilon} / \hbar\right)^{2}},
$$

where the exponential decay originates from the Lorentzian broadening due to electron-phonon coupling and the Gaussian term describes the inhomogeneous broadening caused by charge noise. Notice that $\tau_{\varepsilon}$ is a Fourier variable rather than a real time variable, and thus, $\lambda$ should not be interpreted as physical decay rate. [Only for $\Delta \ll \gamma$, all Lorentzians in Eq. (2) possess the same width, so that $\left.\hat{I}\left(\tau_{\varepsilon}, \tau_{A}\right)\right|_{\text {lemon }}$ is described by Eq. (4) with simply $\lambda=\gamma$ as suggested in Ref. [32].] In Figs. 4(a) and 4(c) we plot measured and calculated decays (dots), respectively, for various temperatures between 18 and $500 \mathrm{mK}$. The solid lines in panels (a) and (c) are identical and express Eq. (4) with $\lambda$ as a fit parameter, while $\lambda^{\star}$ is kept fixed at $3.5 \mu \mathrm{eV}$. Figure 4(b) compares $\lambda(T)$ obtained by this procedure from our measurements (black dots) with the numerical results using three different values of $\alpha_{Z}$. An outstanding agreement between theory and experiments is found at $\alpha_{Z} \simeq 1.5 \times 10^{-4}$ [blue in Fig. 4(b)]. This completes our set of model parameters needed to calculate LZSM patterns as in Figs. 2(c) and 2(d). $\lambda(T)$ increases linearly for $T \gtrsim 100 \mathrm{mK}$, whereas it is bounded by $\lambda_{\min } \simeq 4 \mu \mathrm{eV}$ at our lowest temperatures. This bound marks the intrinsic decay of $\left.\hat{I}\left(\tau_{\varepsilon}, \tau_{A}\right)\right|_{\text {lemon }}$ present even in the low-temperature limit of our transport measurement but is not related to the lowtemperature bound of the qubit coherence time $T_{2}$.

To actually identify $T_{2}(T)$, we use its dependence on $\alpha_{Z}$ in the spin-boson model. In the absence of $\mathrm{rf}$ modulation, it provides the analytical prediction [33]

$$
T_{2}\left(T, \alpha_{Z}\right)=\frac{\hbar}{\pi \alpha_{Z}}\left[\frac{2 k_{B} T \bar{\varepsilon}^{2}}{E^{2}}+\frac{\Delta^{2}}{2 E} \operatorname{coth}\left(\frac{E}{2 k_{B} T}\right)\right]^{-1} .
$$

In the low-temperature limit $k_{B} T \ll E=\sqrt{\Delta^{2}+\bar{\varepsilon}^{2}}$ our undriven qubit has $T_{2}=2 \hbar E / \pi \alpha_{Z} \Delta^{2}$. Assuming $\bar{\varepsilon}=0$, we find $T_{2} \simeq 0.2 \mu \mathrm{s}$, which further increases at finite detuning. Alternatively, $T_{2}$ could be increased by decreasing $\Delta$. This would, however, reduce the clock speed of the qubit. In the same spirit, the rf-induced renormalization of $\Delta \rightarrow \Delta_{n}$ stabilizes the qubit's coherence on the expense of a larger gate operation time [34].

Summarizing, we demonstrated that steady-state LZSM interferometry is a viable tool to fully characterize a qubit including its coupling to a noisy environment. The quantitative agreement between our experiments and our complete system-bath model analyzed with Floquet transport theory allows us to trace the origins of inhomogeneous broadening and decoherence. Thereby we determined the individual values of $T_{2}^{\star}=\hbar / \lambda^{\star}$ and $T_{2}$ of the qubit. Our steady-state method is remarkably simple compared to the alternative pulsed gate experiments. Our two-electron charge qubit is affected by slow charge noise limiting $T_{2}^{\star}$ to $\simeq 0.2 \mathrm{~ns}$ but a coherence time of $T_{2} \simeq 0.2 \mu \mathrm{s}$, being much longer than previously reported values in quantum dot charge qubits $[17,35,36]$. The clock speed of our qubit, $\Delta / h \simeq 3.1 \mathrm{GHz}$, which limits $T_{2}$ at $T \simeq 20 \mathrm{mK}$ and $\bar{\varepsilon}=0$, would then provide enough time for $>600$ quantum operations. At higher temperatures or sizable $\bar{\varepsilon}$, decoherence is dominated by the electron-phonon coupling. Our method is simple, very general, and can be applied to arbitrary qubit systems. An extension including individually controlled Landau-Zener transitions and a combination with nonadiabatic pulses will open up alternative means of quantum information processing. Our two-electron qubit experiments illustrate an interesting approach for studying the interaction of qubits and complex many body quantum systems.

We wish to thank R. Blattmann, E. Hoffmann, M. Kiselev, J. Kotthaus, and P. Nalbach for valuable 
discussions and are grateful for financial support from the DFG via SFB-631 and the Cluster of Excellence "Nanosystems Initiative Munich (NIM)" and by the Spanish Ministry of Economy and Competitiveness through Grant No. MAT2011-24331. S. L. acknowledges support via a Heisenberg fellowship of the DFG. F. F. and G. P. contributed equally to this work.

[1] L. D. Landau, Phys. Z. Sowjetunion 2, 46 (1932).

[2] C. Zener, Proc. R. Soc. A 137, 696 (1932).

[3] E. C. G. Stueckelberg, Helv. Phys. Acta 5, 369 (1932).

[4] E. Majorana, Nuovo Cimento 9, 43 (1932).

[5] W. D. Oliver, Y. Yu, J. C. Lee, K. K. Berggren, L. S. Levitov, and T. P. Orlando, Science 310, 1653 (2005).

[6] M. Sillanpää, T. Lehtinen, A. Paila, Y. Makhlin, and P. Hakonen, Phys. Rev. Lett. 96, 187002 (2006).

[7] C. M. Wilson, T. Duty, F. Persson, M. Sandberg, G. Johansson, and P. Delsing, Phys. Rev. Lett. 98, 257003 (2007).

[8] D. M. Berns, M. S. Rudner, S. O. Valenzuela, K. K. Berggren, W. D. Oliver, L. S. Levitov, and T. P. Orlando, Nature (London) 455, 51 (2008).

[9] S. N. Shevchenko, S. Ashhab, and F. Nori, Phys. Rep. 492, 1 (2010).

[10] J. Stehlik, Y. Dovzhenko, J. R. Petta, J. R. Johansson, F. Nori, H. Lu, and A. C. Gossard, Phys. Rev. B 86, 121303(R) (2012).

[11] E. Dupont-Ferrier, B. Roche, B. Voisin, X. Jehl, R. Wacquez, M. Vinet, M. Sanquer, and S. De Franceschi, Phys. Rev. Lett. 110, 136802 (2013).

[12] G. Petersen, E. A. Hoffmann, D. Schuh, W. Wegscheider, G. Giedke, and S. Ludwig, Phys. Rev. Lett. 110, 177602 (2013).

[13] J. R. Petta, H. Lu, and A. C. Gossard, Science 327, 669 (2010).

[14] P. Huang, J. Zhou, F. Fang, X. Kong, X. Xu, C. Ju, and J. Du, Phys. Rev. X 1, 011003 (2011).

[15] P. Nalbach, J. Knörzer, and S. Ludwig, Phys. Rev. B 87, 165425 (2013).

[16] H. Ribeiro, G. Burkard, J. R. Petta, H. Lu, and A. C. Gossard, Phys. Rev. Lett. 110, 086804 (2013).

[17] G. Cao, H.-O. Li, T. Tu, L. Wang, C. Zhou, M. Xiao, G.-C. Guo, H.-W. Jiang, and G.-P. Guo, Nat. Commun. 4, 1401 (2013).
[18] M. Ciorga, A. S. Sachrajda, P. Hawrylak, C. Gould, P. Zawadzki, S. Jullian, Y. Feng, and Z. Wasilewski, Phys. Rev. B 61, R16315 (2000).

[19] K. Ono, D. G. Austing, Y. Tokura, and S. Tarucha, Science 297, 1313 (2002).

[20] Y. Wang, S. Cong, X. Wen, C. Pan, G. Sun, J. Chen, L. Kang, W. Xu, Y. Yu, and P. Wu, Phys. Rev. B 81, 144505 (2010).

[21] A. M. Satanin, M. V. Denisenko, S. Ashhab, and F. Nori, Phys. Rev. B 85, 184524 (2012).

[22] A. M. Satanin, M. V. Denisenko, A. I. Gelman, and F. Nori, arXiv:1305.4800.

[23] M. N. Kiselev, K. Kikoin, and M. B. Kenmoe, Europhys. Lett. 104, 57004 (2013).

[24] H. Bluhm, S. Foletti, I. Neder, M. Rudner, D. Mahalu, V. Umansky, and A. Yacoby, Nat. Phys. 7, 109 (2011).

[25] See the Supplemental Material at http://link.aps.org/ supplemental/10.1103/PhysRevLett.112.116803 for details.

[26] Y. Kayanuma, Phys. Rev. A 50, 843 (1994).

[27] M. Strass, P. Hänggi, and S. Kohler, Phys. Rev. Lett. 95, 130601 (2005).

[28] G. Granger, D. Taubert, C. E. Young, L. Gaudreau, A. Kam, S. A. Studenikin, P. Zawadzki, D. Harbusch, D. Schuh, W. Wegscheider, Z. R.Wasilewski, A. A. Clerk, S. Ludwig, and A. S. Sachrajda, Nat. Phys. 8, 522 (2012).

[29] A. J. Leggett, S. Chakravarty, A. T. Dorsey, M. P. A. Fisher, A. Garg, and W. Zwerger, Rev. Mod. Phys. 59, 1 (1987).

[30] T. Fujisawa and Y. Hirayama, Appl. Phys. Lett. 77, 543 (2000).

[31] C. Buizert, F. H. L. Koppens, M. Pioro-Ladrière, H.-P. Tranitz, I. T. Vink, S. Tarucha, W. Wegscheider, and L. M. K. Vandersypen, Phys. Rev. Lett. 101, 226603 (2008).

[32] M. S. Rudner, A. V. Shytov, L. S. Levitov, D. M. Berns, W. D. Oliver, S. O. Valenzuela, and T. P. Orlando, Phys. Rev. Lett. 101, 190502 (2008).

[33] U. Weiss and M. Wollensak, Phys. Rev. Lett. 62, 1663 (1989).

[34] K. M. Fonseca-Romero, S. Kohler, and P. Hänggi, Chem. Phys. 296, 307 (2004).

[35] T. Hayashi, T. Fujisawa, H. D. Cheong, Y. H. Jeong, and Y. Hirayama, Phys. Rev. Lett. 91, 226804 (2003).

[36] K. D. Petersson, J. R. Petta, H. Lu, and A. C. Gossard, Phys. Rev. Lett. 105, 246804 (2010). 